\title{
Fermion masses in noncommutative geometry
}

\author{
R. Schelp \\ Department of Physics, University of Texas at Austin, Austin, Texas 78712, USA \\ E-mail: schelp@physics.utexas.edu
}

(August 6, 2018)

\begin{abstract}
Recent indications of neutrino oscillations raise the question of the possibility of incorporating massive neutrinos in the formulation of the Standard Model (SM) within noncommutative geometry (NCG). We find that the NCG requirement of Poincaré duality constrains the numbers of massless quarks and neutrinos to be unequal unless new fermions are introduced. Possible scenarios in which this constraint is satisfied are discussed.
\end{abstract}

PACS Numbers: 12.15.Ff, 14.60.St, 02.40.-k

Keywords: Noncommutative geometry; Neutrino; Mass; Poincaré duality

\section{INTRODUCTION}

The results of recent solar [1], atmospheric [2], and accelerator-based [3] neutrino experiments all suggest that neutrino oscillations do occur. This leads one to the idea that, as in the case for quarks, the neutrino flavor eigenstates are coherent linear superpositions of mass eigenstates. For a neutrino of flavor $\ell$ we have

$$
\left|\nu_{\ell}\right\rangle=\sum_{i=1}^{N} U_{\ell i}\left|\nu_{i}\right\rangle,
$$

where $U_{\ell i}$ is the leptonic mixing matrix. A straightforward quantum mechanical calculation leads to the following expression [4] for the probablility that a neutrino of flavor $\ell$ with a momentum $p_{\nu}$ will oscillate into a neutrino of flavor $\ell^{\prime}$ in a distance $L$ :

$$
\begin{aligned}
P_{\ell \ell^{\prime}}\left(p_{\nu}, L\right) & =\sum_{i=1}^{N}\left|U_{\ell i} U_{i \ell^{\prime}}^{\dagger}\right|^{2} \\
& +\operatorname{Re}\left\{\sum_{\substack{i, j=1 \\
j \neq i}}^{N} U_{\ell i} U_{i \ell^{\prime}}^{\dagger} U_{\ell j} U_{j \ell^{\prime}}^{\dagger} e^{i \frac{\left|m_{i}^{2}-m_{j}^{2}\right| L}{2 p_{\nu}}}\right\},
\end{aligned}
$$

where $m_{i}$ is the mass of the $i$ th mass eigenstate.

In the usual SM, neutrinos are taken to be massless; experiments have only given upper bounds on the masses. However, nothing forces neutrinos to be massless, and if one takes the oscillation experiments seriously, then the analysis above shows that neutrinos must have mass. The simplest way to modify the SM to accomodate massive neutrinos is by including $\nu_{R}$ states and Yukawa couplings for the neutrinos (which would involve the leptonic mixing matrix $U_{\ell i}$ above).

It has been shown that, while a NCG version of the $\mathrm{SM}$ is possible, there are many Yang-Mills-Higgs models which cannot be formulated within the NCG framework [5]. In this article we address the question of the extent to which the NCG formulation of the SM allows for massive neutrinos. This question has been addressed for an earlier NCG version of the SM [6]. Here we reconsider the issue in the context of the requirement of Poincaré duality.

\section{NONCOMMUTATIVE GEOMETRY}

We begin with a brief overview of the main ideas of NCG á la Connes as they pertain to models of particle physics. We refer to [7] for a clear and thorough review of NCG as it is applied to the SM.

The basic data required to specify a NCG are grouped together in a package called a real spectral triple [8]. A spectral triple $(\mathcal{A}, \mathcal{H}, D)$ consists of a Hilbert space $\mathcal{H}$ on which the involutive algebra $\mathcal{A}$ is represented as a subalgebra of $\mathcal{B}(\mathcal{H})$, together with a self-adjoint operator $D$. The operator $D$ need not be bounded, but we require that $[D, a]$ be bounded for all $a \in \mathcal{A}$ and that the resolvent $(D-\lambda)^{-1}, \lambda \notin \mathbb{R}$ of $D$ is compact. If there exists an operator $\gamma$ on $\mathcal{H}$ which satisfies $\gamma^{*}=\gamma, \gamma^{2}=1$, $D \gamma=-\gamma D$, and $\gamma a=a \gamma$ for all $a \in \mathcal{A}$, then we classify $(\mathcal{A}, \mathcal{H}, D)$ as a even spectral triple. A real spectral triple is an even spectral triple for which there also exists an antilinear isometry $J$ which satisfies $J D=D J, J^{2}=\epsilon$, $J \gamma=\epsilon^{\prime} \gamma J$, where $\epsilon$ and $\epsilon^{\prime}$ take values in $\{+1,-1\}$ depending on the dimension of the spectral triple. We also require that both $\left[a, J b J^{*}\right]$ and $\left[[D, a], J b J^{*}\right]$ vanish for all $a, b \in \mathcal{A}$.

The NCG particle physics models which have been constructed thus far all make use of the notion of the product of real spectral triples. One factor of the product encodes the spacetime of the model and the other factor encodes the internal space (the gauge group). For the standard model the components of the spectral triple are

$$
\begin{aligned}
\mathcal{A} & =\mathcal{A}_{\mathrm{S}} \otimes \mathcal{A}_{\mathrm{F}}=C^{\infty}\left(\mathbb{R}^{4}, \mathbb{R}\right) \otimes\left(\mathbb{C} \oplus \mathbb{H} \oplus M_{3}(\mathbb{C})\right), \\
\mathcal{H} & =L^{2}\left(\mathbb{R}^{4}, S\right) \otimes \mathcal{H}_{\mathrm{F}} \\
D & =\not \partial \otimes 1+\gamma_{5} \otimes D_{\mathrm{F}}
\end{aligned}
$$




$$
\begin{aligned}
& \gamma=\gamma_{5} \otimes \gamma_{F}, \\
& J=C \otimes J_{F} .
\end{aligned}
$$

where $\mathcal{H}_{\mathrm{F}}$ is a complex Hilbert space which has a basis labeled by the elementary fermions (including antiparticles), $D_{\mathrm{F}}$ contains the Yukawa couplings, $\gamma_{F}$ is the grading by chirality on $\mathcal{H}_{\mathrm{F}}, C$ is the charge conjugation operator on spinors, and $J_{\mathrm{F}}$ is charge conjugation on $\mathcal{H}_{\mathrm{F}}$.

\section{POINCARÉ DUALITY}

In classical differential geometry, Poincaré duality is the requirement that for $p$-forms $\alpha$ and $\beta$ the scalar product

$$
(\alpha \mid \beta)=\int \alpha \wedge * \beta
$$

vanishes for all $\beta$ only if $\alpha=0$. Connes has put forth seven axioms defining NCGs [9] of which Riemannian geometries are then seen as special cases. The application of Connes' generalization of the Poincaré duality condition to zero-dimensional spectral triples (ones based on matrix algebras) is nicely explained in [7]. Here we generalize the NCG formulation of the SM to allow for both right-handed neutrinos and charge-2/3 quarks without right-handed states and derive the implications of Poincaré duality in this setting.

Our first step is to adjust the representation of the algebra $\mathcal{A}_{\mathrm{F}}=\mathbb{C} \oplus \mathbb{H} \oplus M_{3}(\mathbb{C})$. Denote an element of $\mathcal{A}_{\mathrm{F}}$ by the triple $(\lambda, q, m)$, where $\lambda \in \mathbb{C}, q \in \mathbb{H}$, and $m \in M_{3}(\mathbb{C})$. Recall also that a quaternion may be expressed as $q=\alpha+\beta j$, where $\alpha, \beta \in \mathbb{C}$. For $N_{1}$ generations of leptons without right-handed neutrinos, the representation on the particle space is

$$
\begin{aligned}
& \begin{array}{llll}
\ell_{R} & \nu_{R} & \ell_{L} & \nu_{L}
\end{array} \\
& \pi_{\ell 1}^{+}(\lambda, q)=\begin{array}{c}
\ell_{R} \\
\nu_{R} \\
\ell_{L} \\
\nu_{L}
\end{array}\left(\begin{array}{cccc}
\lambda & & & \\
& 0 & & \\
& & \alpha & \beta \\
& & -\bar{\beta} & \bar{\alpha}
\end{array}\right) \otimes \mathbb{1}_{N_{1}} .
\end{aligned}
$$

In the remaining $N-N_{1}$ generations we include righthanded neutrinos, thus the appropriate representation is

$$
\pi_{\ell 2}^{+}(\lambda, q)=\begin{gathered}
\ell_{R} \\
\ell_{R} \\
\nu_{R} \\
\ell_{L} \\
\nu_{L}
\end{gathered}\left(\begin{array}{cccc}
\lambda & \nu_{R} & \ell_{L} & \nu_{L} \\
& \bar{\lambda} & & \\
& & \alpha & \beta \\
& & -\bar{\beta} & \bar{\alpha}
\end{array}\right) \otimes \mathbb{1}_{N-N_{1}} .
$$

In the above, $\ell$ denotes $e, \mu$, or $\tau$, not the weak doublets. For the quarks, the representations are the same except that we replace $N_{1}$ by $N_{2}$, the number of generations for which the charge- $2 / 3$ quark has no right-handed state, and tensor each of the above by $\mathbb{1}_{3}$ for color. We must also give the representations of $(\lambda, q, m)$ on the antiparticles, which are simply multiplication by $\bar{\lambda}$ for the leptons and multiplication by $m$ for the quarks.
The total Hilbert space of the theory is

$$
\mathcal{H}=\mathcal{H}_{\ell}^{+} \oplus \mathcal{H}_{q}^{+} \oplus \mathcal{H}_{\ell}^{-} \oplus \mathcal{H}_{q}^{-}
$$

where

$$
\begin{aligned}
\mathcal{H}_{\ell}^{+} & =\mathbb{C}_{R} \otimes \mathbb{C}^{N_{1}} \oplus \mathbb{C}_{L}^{2} \otimes \mathbb{C}^{N_{1}} \\
& \oplus(\mathbb{C} \oplus \mathbb{C})_{R} \otimes \mathbb{C}^{N-N_{1}} \oplus \mathbb{C}_{L}^{2} \otimes \mathbb{C}^{N-N_{1}}
\end{aligned}
$$

for the leptons and

$$
\begin{aligned}
& \mathcal{H}_{q}^{+}=\mathbb{C}_{R} \otimes \mathbb{C}^{N_{2}} \otimes \mathbb{C}_{\mathrm{col}}^{3} \oplus \mathbb{C}_{L}^{2} \otimes \mathbb{C}^{N_{2}} \otimes \mathbb{C}_{\mathrm{col}}^{3} \\
& \oplus(\mathbb{C} \oplus \mathbb{C})_{R} \otimes \mathbb{C}^{N-N_{2}} \otimes \mathbb{C}_{\mathrm{col}}^{3} \oplus \mathbb{C}_{L}^{2} \otimes \mathbb{C}^{N-N_{2}} \otimes \mathbb{C}_{\mathrm{col}}^{3}
\end{aligned}
$$

for the quarks. $\mathcal{H}_{\ell}^{-}$and $\mathcal{H}_{q}^{-}$are the corresponding antiparticle Hilbert spaces.

The Poincaré duality condition is rooted in $K$-theory. For a zero-dimensional spectral triple the condition amounts to the nondegeneracy of the intersection form

$$
Q_{i j}=\left(p_{i}, p_{j}\right)=\operatorname{Tr}\left(\gamma p_{i} J p_{j} J^{*}\right),
$$

where $\gamma$ is the chirality operator, $J$, the charge conjugation operator, is the real structure on the spectral triple, and the $p_{i}$ s are generators of $K_{0}(\mathcal{A})$. For the finite part of the algebra we are using, $K_{0}\left(\mathcal{A}_{\mathrm{F}}\right)=\mathbb{Z} \oplus \mathbb{Z} \oplus \mathbb{Z}$. This group is generated by the minimal-rank projections 1 for $\mathbb{C}, \mathbb{1}_{2}$ for $\mathbb{H}$, and $e=\operatorname{diag}(1,0,0)$ for $M_{3}(\mathbb{C})$.

For our calculations we choose a basis in which $p_{1}=$ $(-1) \oplus e, p_{2}=1 \oplus \mathbb{1}_{2}$, and $p_{3}=1$. In terms $N_{1}$ and $N_{2}$ defined above, the chirality and projections take the form

$$
\begin{aligned}
& \gamma(1,0,-1,-1)^{N_{1}} \oplus(1,1,-1,-1)^{N-N_{1}} \\
& \oplus(1,0,-1,-1)^{3 N_{2}} \oplus(1,1,-1,-1)^{3\left(N-N_{2}\right)} \\
& \oplus(1,0,-1,-1)^{N_{1}} \oplus(1,1,-1,-1)^{N-N_{1}} \\
& \oplus(1,0,-1,-1)^{3 N_{2}} \oplus(1,1,-1,-1)^{3\left(N-N_{2}\right)}, \\
& p_{1} \mapsto(-1,0,0,0)^{N_{1}} \oplus(-1,-1,0,0)^{N-N_{1}} \\
& \oplus(-1,0,0,0)^{3 N_{2}} \oplus(-1,-1,0,0)^{3\left(N-N_{2}\right)} \\
& \oplus(-1,0,-1,-1)^{N_{1}} \oplus(-1,-1,-1,-1)^{N-N_{1}} \\
& \oplus(e, 03, e, e)^{N_{2}} \oplus(e, e, e, e)^{N-N_{2}}, \\
& p_{2} \mapsto(1,0,1,1)^{N_{1}} \oplus(1,1,1,1)^{N-N_{1}} \\
& \oplus(1,0,1,1)^{3 N_{2}} \oplus(1,1,1,1)^{3\left(N-N_{2}\right)} \\
& \oplus(1,0,1,1)^{N_{1}} \oplus(1,1,1,1)^{N-N_{1}} \\
& \oplus(0,0,0,0)^{3 N_{2}} \oplus(0,0,0,0)^{3\left(N-N_{2}\right)}, \\
& p_{3} \mapsto(1,0,0,0)^{N_{1}} \oplus(1,1,0,0)^{N-N_{1}} \\
& \oplus(1,0,0,0)^{3 N_{2}} \oplus(1,1,0,0)^{3\left(N-N_{2}\right)} \\
& \oplus(1,0,1,1)^{N_{1}} \oplus(1,1,1,1)^{N-N_{1}} \\
& \oplus(0,0,0,0)^{3 N_{2}} \oplus(0,0,0,0)^{3\left(N-N_{2}\right)} .
\end{aligned}
$$


These projections can be read off from the representations given above, taking $(\lambda, q, m)$ to be $(-1,0, e)$ for $p_{1}$, $\left(1, \mathbb{1}_{2}, 0\right)$ for $p_{2}$, and $(1,0,0)$ for $p_{3}$.

In order to calculate $Q$ we note that $J_{\mathrm{F}}$ acts by

$$
J_{\mathrm{F}}\left(\begin{array}{c}
\xi \\
\bar{\eta}
\end{array}\right)=\left(\begin{array}{c}
\eta \\
\bar{\xi}
\end{array}\right),
$$

where $(\xi, \bar{\eta}) \in \mathcal{H}^{+} \oplus \mathcal{H}^{-}$. The effect on $p_{i}$ of conjugation by $J_{\mathrm{F}}$ is therefore to interchange the first four terms in the direct sum with the last four terms.

Because the application of NCG methods to particle physics is relatively new, we include here the details of the calculation of $Q_{12}$. We work out the argument of the trace in two pieces. The first factor is

$$
\begin{aligned}
\gamma p_{1} \mapsto & (-1,0,0,0)^{N_{1}} \oplus(-1,-1,0,0)^{N-N_{1}} \\
& \oplus(-1,0,0,0)^{3 N_{2}} \oplus(-1,-1,0,0)^{3\left(N-N_{2}\right)} \\
& \oplus(-1,0,1,1)^{N_{1}} \oplus(-1,-1,1,1)^{N-N_{1}} \\
& \oplus\left(e, 0_{3},-e,-e\right)^{N_{2}} \oplus(e, e,-e,-e)^{N-N_{2}}
\end{aligned}
$$

and the second factor is

$$
\begin{aligned}
J p_{2} J^{*} \mapsto & (1,0,1,1)^{N_{1}} \oplus(1,1,1,1)^{N-N_{1}} \\
& \oplus(0,0,0,0)^{3 N_{2}} \oplus(0,0,0,0)^{3\left(N-N_{2}\right)} \\
& \oplus(1,0,1,1)^{N_{1}} \oplus(1,1,1,1)^{N-N_{1}} \\
& \oplus(1,0,1,1)^{3 N_{2}} \oplus(1,1,1,1)^{3\left(N-N_{2}\right)}
\end{aligned}
$$

The product of these two factors is

$$
\begin{aligned}
\gamma p_{1} J p_{2} J^{*} & \mapsto(-1,0,0,0)^{N_{1}} \oplus(-1,-1,0,0)^{N-N_{1}} \\
& \oplus(0,0,0,0)^{3 N_{2}} \oplus(0,0,0,0)^{3\left(N-N_{2}\right)} \\
& \oplus(-1,0,1,1)^{N_{1}} \oplus(-1,-1,1,1)^{N-N_{1}} \\
& \oplus(e, 0,-e,-e)^{N_{2}} \oplus(e, e,-e,-e)^{N-N_{2}} .
\end{aligned}
$$

The trace of this product is

$$
\begin{aligned}
\operatorname{Tr}\left(\gamma p_{1} J p_{2} J^{*}\right) & =-N_{1}-2\left(N-N_{1}\right)+N_{1}-N_{2} \\
& =-2 N+2 N_{1}-N_{2} .
\end{aligned}
$$

Performing similar calculations for the remaining elements, we find the intersection form $Q$, omitting an overall factor of -2 , to be

$$
\left(\begin{array}{ccc}
N_{1}-N_{2} & N-N_{1}+\frac{1}{2} N_{2} & N-N_{1}+\frac{1}{2} N_{2} \\
N-N_{1}+\frac{1}{2} N_{2} & N_{1} & N_{1}-N \\
N-N_{1}+\frac{1}{2} N_{2} & N_{1}-N & N_{1}-2 N
\end{array}\right) .
$$

First of all, we note that for $N_{1}=N$ neutrinos without right-handed states and $N_{2}=0$ quarks lacking righthanded states, as is the case in the SM, we get

$$
Q=2 N\left(\begin{array}{ccc}
-1 & 0 & 0 \\
0 & -1 & 0 \\
0 & 0 & 1
\end{array}\right)
$$

which agrees with the result of 8 . The determinant of this matrix is $8 N^{3}$, so it is invertible and Poincaré duality condition is satisfied.

If we keep right-handed states for all quarks (again, $N_{2}=0$ ) and let $N_{1}=0$ so as to build an extension of the $\mathrm{SM}$ in which all neutrinos are massive, the intersection form is

$$
Q=2 N\left(\begin{array}{ccc}
0 & -1 & -1 \\
-1 & 0 & 1 \\
-1 & 1 & 2
\end{array}\right)
$$

recovering the result of Testard, as reported in [7]. Here the determinant of $Q$ vanishes, indicating that the Poincaré duality condition is not satisfied in this case.

In the general case we find that $\operatorname{det} Q=8\left(N_{1}-N_{2}\right) N^{2}$. The requirement that $Q$ be nondegenerate necessitates that the determinant not vanish. Thus, we must have $N_{1} \neq N_{2}$.

\section{POSSIBLE SOLUTIONS}

There are three ways to satisfy this constraint while incorporating massive neutrinos: (1) Keep all of the righthanded quark states $\left(N_{2}=0\right)$ and let one of the neutrinos (presumably $\nu_{e}$ ) remain massless $\left(N_{1}=1\right) ;(2)$ Give right-handed states to all of the neutrinos $\left(N_{1}=0\right)$ and let the up quark remain massless (so that $N_{2}=1$ ); (3) Give masses to all quarks and leptons $\left(N_{1}=N_{2}=0\right)$ and introduce new fermions (perhaps a sterile neutrino). We now explore these possibilities.

1. No massless quarks, one massless neutrino. The conventional wisdom with regard to neutrino oscillations is that neutrino masses are necessary in order for them to occur. In the standard analysis given in the introduction, though, only mass differences play a role, allowing for the possibility of oscillations between massless and massive neutrinos. Many more sophisticated treatments of neutrino oscillations have been carried out (see the references in [10]), but it seems that in general they do not rely on neutrino masses, but mass differences to generate oscillations.

With this in mind, we consider a case in which the eigenvalue of the first of the three mass eigenstates is zero. In this case there are three mass-squared differences, $m_{12}^{2}, m_{23}^{2}$, and $m_{31}^{2}$, which must sum to zero (here $m_{i j}^{2}=m_{i}^{2}-m_{j}^{2}$, where $m_{i}$ is the eigenvalue of the $i$ th mass eigenstate), leaving two independent masses. Setting $m_{1}=0$ fixes the overall scale of the masses, but still leaves two independent parameters. By adjusting $m_{2}$ and $m_{3}$ we can account for two different oscillation lengths; just as is the case were we to add neutrino masses to the SM apart from the NCG context. With just two mass scales, we cannot explain the results of all three types of neutrino oscillation experiments mentioned in the introduction, but we reserve judgement on this possibility until more experimental verification is available. 
2. One massless quark, no massless neutrinos. Our analysis has shown that Poincaré duality requires that $N_{1} \neq N_{2}$, so we may take all neutrinos to be massive if we are willing to take $m_{u}=0$ (in disagreement with the claim of [7] that the $m_{u}=0$ possibility is excluded). There has been quite a bit of discussion in the literature regarding the mass of the $u$-quark and the possibility of it vanishing (e.g. 11 13). From the standpoint of the strong CP problem, this option is quite attractive. It would lead to a solution in which the determinant of the quark mass matrix vanishes [14,15]. It seems that there is currently no phenomenological result which necessarily excludes the possibility of $m_{u}=0$.

For us, though, the masslessness of the $u$-quark comes from the absence of the right-handed component, not simply from the vanishing of the appropriate Yukawa coupling. This poses a problem for strong interation phenomenology, since it forces the couplings to right-handed and left-handed quarks to be different and would thus lead to parity violation in the strong interactions.

3. New fermions. If we take all of the neutrino experiments seriously, it seems that not even three massive neutrinos are enough to explain via oscillations all of the observed effects [16]. One possible solution is then to include a new neutrino in the model. This option is severely limited by the experimental measurement of the $Z$ width, which is consistent with $2.993 \pm 0.011$ light $\left(2 m_{\nu}<m_{Z}\right)$ neutrino types [17. We can avoid conflict with this result, though, by introducing a neutrino which does not couple in the usual way to the weak gauge bosons - a type of 'sterile' neutrino, as was introduced in [18,19. The effects of such an inclusion would depend on the details of the model and the resulting modification to the matrix $Q$. It has been shown in 20] that the reality axiom of NCG disallows the inclusion of Majorana fermions, thus excluding many of the sterile neutrino models which have been studied. The inclusion of other fermions in general could also provide a solution, depending on the representations in which they appear.

\section{CONCLUSION}

In its NCG setting, the SM, while quite constrained [21], does allow for certain extensions which include righthanded neutrinos and therefore neutrino masses and oscillations. Our analysis of the Poincaré duality condition has shown that, if we allow only the addition of right-handed neutrinos, the number of quarks with righthanded states must be different from the number of neutrinos with right-handed states.

While the option of having a quark without a righthanded state may have been appealing from the point of view of the strong $\mathrm{CP}$ problem, it runs into trouble with regard to parity violation in the strong interactions. The case in which two neutrinos are given right-handed states allows for two independent oscillation lengths, based on our argument that neutrino oscillations may occur between massive and massless eigenstates. The sterile neutrino solution is an interesting one which should be explored further in the context of NCG.

We should also mention that the almost commutative structure that we have used here, where the noncommutativity only appears in the finite part of the algebra, may have to be abandoned at energies near the electroweak scale. Then, if all three neutrinos are found to be massive, the resulting violation of Poincaré duality could be viewed as a signal that the tensor product structure of the spectral triple on which the current NCG realization of the SM is based is a kind of low-energy limit of a 'truly noncommutative' spectral triple. In that case the Poincaré duality condition would have to be reevaluated for the spectral triple from which the one we use today descends.

[1] T. A. Kirsten, Rev. Mod. Phys. 71 (1999) 1213.

[2] M. Shiozawa, Nulc. Instr. and Meth. A 433 (1999) 307.

[3] K. Eitel, New J. Phys. 2 (2000) 1.

[4] F. Boehm and P. Vogel, Physics of Massive Neutrinos, second edition (Cambridge University, Cambridge, 1992) p. 91.

[5] B. Iochum and T. Schücker, Commun. Math. Phys. 178 (1996) 1.

[6] J. M. Gracia-Bondía, Phys. Lett. B 351 (1995) 510.

[7] C. P. Martin, J. M. Gracia-Bondía, and J. C. Várilly, Phys. Rep. 294 (1998) 363.

[8] A. Connes, J. Math. Phys. 36 (1995) 6194.

[9] A. Connes, Commun. Math. Phys. 182 (1996) 155.

[10] B. Kayser, Euro. Phys. J. C3 (1998) 307.

[11] D. B. Kaplan and A. V. Manohar, Phys. Rev. Lett. 56 (1986) 2004.

[12] J. F. Donoghue and D. Wyler, Phys. Rev. D 45 (1992) 892.

[13] T. Banks, Y. Nir, and N. Seiberg, hep-ph/9403203.

[14] R. D. Peccei, in: CP Violation, ed. C. Jarlskog (World Scientific, Singapore, 1989) p. 503.

[15] H.-Y. Cheng, Phys. Rep. 158 (1988) 1.

[16] K. Zuber, Phys. Rep. 305 (1998) 295.

[17] D. Karlen, Euro. Phys. J. C3 (1998) 319.

[18] D. O. Caldwell and R. N. Mohapatra, Phys. Rev. D 48 (1993) 3259.

[19] J. T. Peltoniemi and J. W. F. Valle, Nucl. Phys. B 406 (1993) 409.

[20] T. Krajewski, J. Geom. Phys. 28 (1998) 1.

[21] R. Brout, Nucl. Phys. Proc. Suppl. 65 (1998) 3. 